\title{
Contribuições do enfermeiro para o apoio matricial em saúde mental na atenção básica*
}

Nurses' contributions to matrix support in mental health in primary health care

Aportes del profesional de Enfermería al apoyo matricial en salud mental en la atención básica

\section{Guilherme Emanuel Weiss Pinheiro ${ }^{\mathrm{I}}$, Luciane Prado Kantorski ${ }^{\mathrm{II}}$}

Resumo: Objetivo: identificar as contribuições do enfermeiro no contexto do apoio matricial em saúde mental na atenção básica. Método: pesquisa qualitativa, com base na Avaliação de Quarta Geração, realizada com trabalhadores dos núcleos de apoio matricial e das equipes de referência de um município do sul do Brasil. Foram realizadas 84 horas de observação, 15 entrevistas e dois grupos de validação dos dados, entre dezembro de 2018 e fevereiro de 2019. A análise ocorreu pelo método comparativo constante. Resultados: o enfermeiro contribui com o apoio matricial à medida que atua na inclusão dos núcleos junto às equipes, no exercício do gerenciamento e da liderança, do cuidado compartilhado e na mediação das ações de saúde mental no território. Conclusão: a liderança do enfermeiro é decisiva, pois ao concentrar funções de gerenciamento e assistência, colabora na construção de alternativas de cuidado às pessoas em sofrimento mental na atenção básica.

Descritores: Enfermagem; Saúde mental; Atenção primária à saúde; Gestão em saúde; Assistência integral à saúde

\begin{abstract}
Objective: to identify the nurses' contributions in the context of matrix support in mental health in primary health care. Method: a qualitative research study, based on the Fourth Generation Assessment, carried out with workers from matrix support centers and reference teams of a municipality in southern Brazil. 84 observation hours of, 15 interviews and two groups of data validation were carried out between December 2018 and February 2019. The analysis was performed using the constant comparative method. Results: nurses contribute with matrix support as they act in the inclusion of the nuclei with the teams, in the exercise of management, leadership and shared care, and in the mediation of the mental health actions in the territory. Conclusion: nurses' leadership is decisive, since by concentrating managerial and assistance functions, they collaborate in the construction of care alternatives for people with mental distress in primary health care.
\end{abstract}

Descriptors: Nursing; Mental health; Primary health care; Health management; Comprehensive health care

Resumen: Objetivo: identificar los aportes del profesional de Enfermería en el contexto del apoyo matricial en salud mental en la atención básica. Método: investigación cualitativa, basada en la Evaluación de Cuarta Generación, realizada

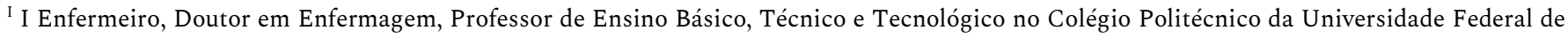
Santa Maria, Santa Maria, Rio Grande do Sul, Brasil. E-mail: enfermeiro.guipinheiro@gmail.com Orcid: http://orcid.org/0000-0003-0069-7023

II Enfermeira, Doutora em Enfermagem, Professora Titular da Faculdade de Enfermagem da Universidade Federal de Pelotas, Pelotas, Rio Grande do Sul, Brasil. E-mail: kantorski@uol.com.br Orcid: http://orcid.org/0000-0001-9726-3162
}

* Extraído da tese “Avaliação de experiências de apoio matricial em saúde mental de uma região de saúde do Rio Grande do Sul”, Programa de Pós-Graduação em Enfermagem, Universidade Federal de Pelotas, 2020. 
Contribuições do enfermeiro para o apoio matricial em saúde mental na atenção básica $\mid 2$

con trabajadores de los centros de apoyo matricial y de los equipos de referencia de un municipio del sur de Brasil. Se realizaron 84 horas de observación, 15 entrevistas y dos grupos de validación de los dados, entre diciembre de 2018 y febrero de 2019. El análisis se realizó por medio del método comparativo constante. Resultados: el profesional de Enfermería contribuye al apoyo matricial en la medida en que se desempeña en la inclusión de los centros junto a los equipos, en el ejercicio de la gestión y del liderazgo, de la atención compartid, y en la mediación de las acciones de salud mental en el territorio. Conclusión: el liderazgo del profesional de Enfermería es decisivo, puesto que, al concentrar funciones de gestión y asistencia, colabora en la construcción de alternativas de atención a las personas con padecimientos mentales en la atención básica.

Descriptores: Enfermería; Salud mental; Atención primaria de salud; Gestión en salud; Atención integral de salud

\section{Introdução}

A Organização Mundial de Saúde estima que, no ano de 2020, no mundo somam-se mais de 27,9 milhões de trabalhadores de enfermagem. Eles correspondem ao maior número de profissionais no setor da saúde, representando aproximadamente $59 \%$ do contingente de pessoal. ${ }^{1}$ Portanto, tais profissionais são responsáveis pela sustentabilidade de diferentes sistemas universais, por exemplo no Brasil, no Reino Unido, no Canadá, na Dinamarca, entre outros países.

A enfermagem brasileira conta com aproximadamente 1,8 milhões de trabalhadores, constituindo-se como categoria que agrega mais da metade da força de trabalho no setor saúde. ${ }^{2-3}$ Na realidade do Sistema Único de Saúde (SUS), é possível notar situação similar: população de aproximadamente 886.309 profissionais, sendo 259.434 enfermeiros (as), 451.200 técnicos (as) em enfermagem e 175.675 auxiliares de enfermagem, representando mais de $50 \%$ da força de trabalho. ${ }^{3}$

O profissional da enfermagem é um dos elementos chaves na sustentação dos sistemas de saúde, uma vez que se trata da profissão que agrega de forma substancial a prática do cuidado. ${ }^{4}$ Este último é considerado como uma atitude de relação comprometida, harmoniosa e protetora das diferentes realidades, social, pessoal e ambiental. $\mathrm{O}$ ato de cuidar significa acompanhar, "estar com”, responsabilizar-se, dar atenção, bom trato, desvelo, acolher e respeitar. ${ }^{5}$

O enfermeiro vem sendo decisivo no atual contexto de saúde, devido às competências desenvolvidas e aprimoradas pela profissão, haja vista a necessidade de considerar - no processo de cuidado - os determinantes sociais, as iniquidades, o envelhecimento da população, ${ }^{4}$ o 
desenvolvimento de condições crônicas, além de outras peculiaridades. Entre as condições crônicas, destaca-se o sofrimento mental. Estudo internacional aponta que os cuidados compartilhados, ou seja, articulados entre profissionais generalistas e especialistas tendem a melhorar os resultados clínicos na assistência a pessoas com depressão e sugere que esta abordagem aperfeiçoe a gestão do cuidado no contexto das condições crônicas. ${ }^{6}$

Ao observar a complexidade da questão do sofrimento mental e a necessidade de articulação de estratégias de cuidado na atenção básica, é preciso refletir sobre o fazer do enfermeiro nesse contexto, o qual precisa ser desenvolvido, qualificado e transformado. Na realidade da Estratégia Saúde da Família (ESF), o enfermeiro assume funções como: assistência direta à população, por meio do acolhimento e da consulta de enfermagem, por exemplo; gerenciamento de serviços; coordenação de programas; construção de relatórios; e abastecimento de sistemas de informação. Muitas dessas atribuições são compartilhadas com os demais trabalhadores da equipe. ${ }^{7-8}$

Nessa perspectiva, destaca-se a importância do trabalho interdisciplinar e interprofissional, pois nenhuma categoria profissional é capaz de centralizar o cuidado integral, sendo necessária a articulação com os demais campos do saber. Com isso, surge a necessidade de ações em saúde mental no contexto da atenção básica serem desenvolvidas com suporte do apoio matricial, que é uma estratégia que ampara os trabalhadores, as pessoas em sofrimento mental, as suas famílias e as comunidades no cuidado, na promoção e na reabilitação da saúde. ${ }^{9}$

O apoio matricial busca a integração dos profissionais especialistas - também conhecidos como apoiadores - com profissionais generalistas, membros das equipes de referência, por meio de práticas interdisciplinares que auxiliam na ampliação da clínica, da qualidade da assistência e na resolutividade dos serviços. ${ }^{10}$ Além da integração dos profissionais, o apoio matricial também agrega elementos do cuidado compartilhado, como suporte educacional, cuidado especializado, regulação, cogestão cuidado multiprofissional, comunicação sistemática, cuidado estruturado e suporte organizacional. 6,11 
Contribuições do enfermeiro para o apoio matricial em saúde mental na atenção básica | 4

No contexto brasileiro, o apoio matricial transformou as metodologias de trabalho em saúde, incluindo elementos como o diálogo, a responsabilização e a decisão coletiva nas ações e relações das e entre as equipes de referência e de apoio matricial. Isso promoveu a ampliação da comunicação entre os trabalhadores que, ao conduzir casos conjuntamente, vivenciam aspectos do cuidado compartilhado no território. ${ }^{12}$

Nesse prisma, o apoio matricial é entendido como uma forma de realizar a atenção à saúde de maneira compartilhada, com o objetivo de prestar cuidado integral e resolutivo, por meio do trabalho interdisciplinar e colaborativo. ${ }^{13}$ Nesse cenário, o enfermeiro é um articulador dessas ações e, muitas vezes, responsável pelo gerenciamento dos serviços e do cuidado.

Entretanto, na literatura existe uma lacuna em relação ao trabalho do enfermeiro com o apoio matricial. A exemplo, uma revisão integrativa demonstra que a maioria dos estudos investigam o matriciamento a partir do trabalho interdisciplinar e não da enfermagem ou de algum campo profissional específico. ${ }^{14}$ Uma revisão de literatura sistemática indica que existe um pequeno número de evidências sobre as intervenções em saúde mental realizadas por enfermeiros na atenção básica, embora aponte que as ações de enfermagem em saúde mental realizadas na atenção básica contribuem para diminuir, significativamente, os sintomas de depressão e ansiedade. ${ }^{15}$

Diante do exposto, este estudo teve como questão de pesquisa: quais são as contribuições do enfermeiro no contexto do apoio matricial na atenção básica? E como objetivo: identificar as contribuições do enfermeiro no contexto do apoio matricial em saúde mental na atenção básica.

\section{Método}

Trata-se de um estudo de abordagem qualitativa, a partir da perspectiva da Avaliação de Quarta Geração, ${ }^{16}$ desenvolvido em uma pesquisa sobre avaliação de experiências de apoio matricial. A diretriz COREQ (Consolidated Criteria for Reporting Qualitative Research) foi utilizada para orientar a redação deste artigo. 
O referencial teórico-metodológico desta investigação está alicerçado na Avaliação de Quarta Geração, a qual diz respeito a uma alternativa às avaliações tradicionais, diante de sua natureza construtivista e responsiva. Ademais, é caracterizada por ser utilizada para designar uma forma distinta de focalizar o processo avaliativo. Assim, define-se quem são os grupos de interesse e o foco organizacional, buscando o consenso crítico entre esses grupos em relação ao objeto de estudo, por meio do processo hermenêutico-dialético. Os parâmetros e limites da avaliação são determinados pelo processo interativo e de negociação, envolvendo os grupos de interesse, os quais determinam o levantamento das questões para coleta de informações. ${ }^{16}$

O termo "construtivista” designa a metodologia empregada, ou seja, a forma de condução da avaliação, alternativa ao paradigma positivista que, por sua vez, pauta-se na mensuração e na quantificação. A perspectiva em questão é considerada responsiva à medida que responde às questões após a coleta de informação, levando em consideração a visão dos grupos de interesse. ${ }^{16}$

Este estudo ocorreu em duas etapas, a primeira foi denominada como "Caracterização dos Núcleos de Apoio à Atenção Básica - Saúde Mental (NAABs)” de uma região de saúde do Rio Grande do Sul (RS). Ela transcorreu de forma online, via formulários eletrônicos (Google Forms ${ }^{\circledR}$ ), enviados aos profissionais dos referidos núcleos, como uma forma de estabelecer a aproximação com o campo para a pesquisa avaliativa. O referido formulário constitui-se, também, de um questionário de caracterização que teve como objetivo conhecer o trabalho e a estrutura de cada núcleo, a exemplo: composição da equipe; carga horária de trabalho; inserção de ações de saúde mental no território; ações de revisão da prática de encaminhamentos; alternativas com vistas à promoção da saúde; e composição da Rede de Atenção à Saúde.

Esta etapa foi realizada no mês de dezembro de 2018, em uma região de saúde do Estado do RS, composta por onze municípios, destes, somente três mantinham o NAAB. Após essa etapa, os dados produzidos, oriundos do questionário de caracterização, foram analisados considerando os critérios constantes na legislação de criação do núcleo. ${ }^{17}$ Ao final da análise, um município 
Contribuições do enfermeiro para o apoio matricial em saúde mental na atenção básica | 6

(pertencente a região de saúde selecionada para a pesquisa), que atendia aos critérios da normativa: como, composição da equipe, carga horária, trabalho coincidente ao horário das equipes de atenção básica, inserção de ações de saúde mental na lógica do território, revisão da prática de encaminhamentos e ações de promoção à saúde, foi escolhido para participar da próxima etapa.

A segunda etapa, a "Avaliação de Quarta Geração", ocorreu a partir da aproximação do pesquisador principal, por meio de visitas à secretaria municipal de saúde do município escolhido na etapa anterior, entre os meses de janeiro e fevereiro de 2019. Dentre as características do município selecionado, pode se destacar que é de pequeno porte, situado no sul do Brasil, com população estimada de 12.561 habitantes, ${ }^{18}$ cuja rede de saúde era constituída por serviços próprios e conveniados. No que diz respeito à atenção básica, o município mantinha quatro equipes de ESF urbanas e mistas (urbana e rural) e uma equipe de ESF rural. Além disso, possuía dois núcleos de apoio matricial, o NAAB e o Núcleo de Apoio à Saúde da Família (NASF), dois polos de academia da saúde e uma oficina terapêutica, que conta com profissionais da Educação Física e de Música, os quais realizam atividades em parceria com a atenção básica e outros dispositivos do território.

A coleta de dados sucedeu por meio do Círculo Hermenêutico Dialético, o qual consistia em uma metodologia de coleta de dados empíricos. Para tal, os pesquisadores selecionaram um respondente inicial, de forma intencional, sendo um indivíduo que ocupava uma posição estratégica em relação ao objeto da avaliação. Em seguida, os outros trabalhadores foram indicados pelos indivíduos que participavam das entrevistas para compor o referido círculo. ${ }^{16}$

Foram realizadas entrevistas semiestruturadas com trabalhadores, apresentando questões focadas nos aspectos relacionados ao trabalho do apoio matricial, por exemplo: como sucedia, características, dificuldades, facilidades, avaliação dos participantes, dentre outros aspectos. As entrevistas ocorreram em salas, de forma individual e privada, nas unidades de saúde do município, com duração média de 27 minutos, foram gravadas em áudio, transcritas na íntegra e, após, analisadas. Os participantes foram abordados pessoalmente pelos pesquisadores, sendo agendada a 
entrevista, conforme ocorria a indicação. Durante as entrevistas, questões abertas eram realizadas.

Participaram do estudo 15 trabalhadores, os quais eram: sete integrantes dos núcleos de apoio matricial, NAAB e NASF; e, oito integrantes das equipes de ESF. Os participantes eram: enfermeiras, técnicas em enfermagem, agentes comunitárias de saúde, médico, terapeuta ocupacional, artesã, psicólogo, acompanhante terapêutica, fisioterapeuta, nutricionista e fonoaudióloga. Não ocorreu nenhuma recusa de participação dos indicados. Em relação às características dos trabalhadores entrevistados, registra-se que: 13 são do sexo feminino e dois do sexo masculino; nove tinham entre 27 e 39 anos e seis entre 40 e 56 anos; em relação ao tempo de atuação na profissão, dois atuavam há menos de dois anos, sete entre 2 e 10 anos e seis entre 11 e 32 anos. Os critérios de seleção utilizados foram: ser trabalhador(a) de equipe de referência ou de núcleo de apoio do município; e, ter sido indicado no Círculo Hermenêutico Dialético.

Foi realizada observação participante, constituindo-se como uma técnica de estudo da interação social que facilita a observação do agir dos indivíduos, composta por meio do relato de quem participa da interação, assim, a observação participante ocorreu no contexto natural da interação social. ${ }^{19}$ Essa observação é denominada etnografia prévia, ${ }^{16}$ e realizou-se no cotidiano dos serviços de atenção básica, acompanhando o dia a dia dos trabalhadores, em visitas domiciliares, atendimentos individuais e grupais, reuniões e atividades, perfazendo um total de 84 horas, entre janeiro e fevereiro de 2019, tais observações foram registradas em notas de diário de campo.

Ao final das observações e das entrevistas semiestruturadas, ocorreu a negociação por meio dos grupos de validação e negociação, realizado com os trabalhadores, com duração total de três horas, no dia 27 de fevereiro de 2019, na sala de reuniões da secretaria municipal de saúde. Foram organizados dois grupos, um com os trabalhadores do apoio matricial e outro com os das equipes de referência, sendo apresentados os principais resultados do trabalho de campo, sistematizando os consensos e os dissensos, buscando possíveis alternativas, juntamente com os participantes.

A análise de dados procedeu de forma paralela ao processo de coleta, sendo baseada no 
Contribuições do enfermeiro para o apoio matricial em saúde mental na atenção básica $\mid 8$

Método Comparativo Constante, com duas etapas: a identificação das unidades de informação e a construção de núcleos temáticos ou categorização. A primeira consiste em identificar as sentenças ou falas extraídas do material empírico, registradas pelo pesquisador. A segunda diz respeito à busca por categorias provisórias, aproximadas pela semelhança no conteúdo, para após a realização do grupo de validação e negociação, constituir as categorias ou núcleos temáticos definitivos. ${ }^{16}$

Ressalta-se que a pesquisa foi submetida à apreciação no Comitê de Ética em Pesquisa com seres humanos, obtendo Aprovação sob Parecer № 3.038.987, de 26 de novembro de 2018, obtendo Certificado de apresentação para Apreciação Ética, sob o número: 02237118.2.0000.5316. A investigação foi conduzida de acordo com os padrões éticos exigidos na legislação nacional do Ministério da Saúde (Resolução № 466/2012; Resolução №510/2016 e Resolução № 580/2018). Os participantes assinaram o Termo de Consentimento Livre e Esclarecido em duas vias, permanecendo uma com o participante e outra com o pesquisador.

Para preservar a identidade dos participantes, utilizou-se códigos. Para tanto, os trabalhadores dos núcleos de apoio matricial foram identificados com a letra A seguida do número da entrevista (A1-A7), ao passo que os integrantes das equipes de ESF com a letra E seguida do número da entrevista (E1-E8). Os trechos de diário de campo provenientes da observação participante são indicados por DC, seguido do número do diário (DC1-DC16) e do grupo de validação por GVN, indicando o participante (GNV-E1 a GNV-E8 ou GNV-A1 a GNV-A8).

As atividades de pesquisa foram conduzidas pelos autores deste artigo, que possuem formação na área da Enfermagem, além de experiência em estudos qualitativos e em avaliação de serviços. As observações foram realizadas somente pelo pesquisador principal, o qual possui expertise na área. O conhecimento dos participantes acerca dos pesquisadores está associado a interações com profissionais de instituições de ensino, pesquisadores na área da saúde - em que a investigação em pauta se tratava de uma pesquisa de doutorado na área da Enfermagem. As entrevistas e observações foram findadas de acordo com o critério de saturação dos dados. 


\section{Resultados}

O apoio matricial é constituído pelos núcleos NAAB e NASF, os quais oferecem retaguarda assistencial e suporte didático-pedagógico ${ }^{13}$ para quatro ESF do município selecionado para este estudo, sendo que os trabalhadores do matriciamento organizam-se em duplas para acompanhá-las. As duplas são formadas por um integrante de cada núcleo, os quais são responsáveis pelo acompanhamento das equipes. Assim, participam de reuniões tanto no âmbito das unidades de saúde, quanto da gestão municipal, nas quais discutem situações e demandas destacadas no apoio, seja de; planejamento; atividades; contribuição na Educação Permanente em Saúde. É importante assinalar que, por meio dessas ações, formam um elo entre a assistência e a gestão e vice-versa.

Os resultados apresentados neste estudo concentram-se na visão dos trabalhadores de saúde acerca das contribuições do enfermeiro para o apoio matricial e para o cuidado em saúde mental na atenção básica. No período de coleta de dados, as equipes participantes da pesquisa encontravam-se completas, do ponto de vista de composição profissional. Os depoimentos apresentam os enfermeiros facilitadores da entrada do apoio matricial junto às equipes de saúde:

Quando tu consegues ter uma relação, de mostrar teu trabalho para enfermeira, ela acaba te puxando sempre que ela precisa e isso tem acontecido no meu trabalho. No momento, estou encontrando mais dificuldades na equipe [se referiu a uma equipe de referência]. Eu noto que não está acontecendo esse vínculo meu com a equipe e, principalmente, com a enfermeira. Em outros locais sempre aconteceu, me acionavam como se eu fosse parte da equipe, elas tinham confiança em mim, confiavam sabendo que eu ia lidar com a situação. (A6)

$A$ entrada da ESF é sempre a enfermagem, e isso a gente percebe bastante, pois dependendo de quem está na frente as coisas acontecem mais. É que a enfermeira toma para si e dialoga com a equipe e acontece. (A7)

Os trabalhadores do apoio matricial reconhecem o trabalho do enfermeiro e as particularidades de cada um em conduzir a equipe. Assim, avaliam, de certa forma, a atuação desse profissional e realizam comparações. 
Contribuições do enfermeiro para o apoio matricial em saúde mental na atenção básica | 10

Hoje eu estou trabalhando numa equipe que é muito boa, a enfermeira é muito legal, ela tem jeito na conversa, tudo flui, ela tem um jeito de levar as coisas muito bem, então as coisas caminham de forma boa, mas eu já trabalhei em outras equipes que não eram assim, que não tinha essa união. (A2)

A liderança dos enfermeiros junto às equipes é notável e considerada um diferencial, uma vez que atua para a condução do trabalho na atenção básica com vistas a qualificação do cuidado.

A enfermeira é destaque e apresenta postura de liderança diante da equipe, das pessoas assistidas e da gestão, realiza as cobranças necessárias, problematiza os assuntos com sabedoria, e reflete, fazendo autocrítica ao trabalho. (DC11)

Outro aspecto a ser destacado diz respeito à abertura das equipes em relação ao apoio matricial, sendo que os enfermeiros são fundamentais nesse processo. Estes, por sua vez, consideram e tratam o matriciamento como prioridade, em especial com casos complexos ou de saúde mental e nas questões organizativas da unidade.

A enfermagem prioriza bastante a questão do matriciamento, principalmente com aqueles pacientes que tem dificuldade na adesão ao tratamento, casos complexos e quase sempre nos de saúde mental. (A3)

Enfermeira solicita apoio do NAAB e NASF na construção do cronograma de atividades e para planejar algo com as comunidades rurais. (DC11)

Há, também, dificuldades vivenciadas pelos trabalhadores para atuar com questões de saúde mental. Eles apontam a frequência de casos que chegam nas unidades, as dificuldades de encaminhamentos e atendimentos que enfrentam:

É só o que está aparecendo para nós, é só o que está chegando, e mais de um caso no dia, seja psicose aguda, crônica, seja alcoolismo, seja droga, seja o que for [...]. Ah! Não tem uma responsabilização, não tem alguém que tu possas encaminhar, é uma área difícil de trabalhar, porque o familiar vem super abalado. (GVN - E1)

Eu fiquei bem surpresa com a quantidade de casos de saúde mental aqui no município, seja transtorno, seja dependência de substância, são muitos, quase metade da população faz uso de medicação controlada. Então, assim, tu tens 
que dar conta, porque esses pacientes estão todos os dias dentro da unidade e a gente tem que dar conta. (GVN - E3)

Diante das dificuldades enfrentadas, os trabalhadores apontam algumas estratégias a serem construídas como alternativa para superação dos problemas. A Educação Permanente em Saúde é uma das estratégias que o enfermeiro, como membro da equipe identifica para enfrentar as dificuldades, as lacunas de conhecimento e a otimização do trabalho na atenção básica.

Sabe a equipe da atenção básica, enfim, tem que estar sempre construindo conhecimento, tem que estar mais preparada para isso. Acho que, de repente, investir em educação permanente nessa área é bem importante aqui para o município. (GVN - E3)

Outras estratégias são destacadas pelos enfermeiros, dentre elas a construção de vínculos e o reconhecimento da realidade do território para, a partir disso, desenvolver ações de cuidado integral e tomada de decisões assertivas no desenvolvimento do trabalho, liderando a equipe de forma a qualificar a assistência. O envolvimento desse profissional neste processo é destacado:

A enfermeira é envolvida com o trabalho e com a equipe, além de possuir um bom vínculo com a população [...]. Conhecia os usuários pelo nome, sabia a procedência [se vinham de localidades distantes ou não]. É uma pessoa que tem bom conhecimento da realidade do seu território, facilitando o acesso das pessoas ao serviço. Além do mais, apresenta uma boa liderança em relação à equipe, pois os profissionais recorrem a ela em momentos de dúvidas. (DC10)

Os resultados apresentados evidenciam a importância do enfermeiro na articulação com o apoio matricial. Isso ocorre mediante a priorização do profissional de Enfermagem com: trabalho em equipe, seu vínculo com as pessoas assistidas e sua implicação com o território, fortalecendo o cuidado em saúde mental na comunidade.

\section{Discussão}

Os participantes do estudo apontam que o enfermeiro, ao reconhecer o matriciamento, compartilha de conquistas e quando a equipe tem essa compreensão o trabalho flui. Nesse contexto, 
Contribuições do enfermeiro para o apoio matricial em saúde mental na atenção básica | 12

os depoimentos dos entrevistados ressaltam a presença do profissional em questão como uma figura importante, que agrega, fazendo que suas atividades laborais ocorram de forma conjunta.

O fato de o enfermeiro acreditar no papel do apoio matricial e colaborar em todo o processo, pode estar associado à concentração de funções de gerenciamento em seu fazer cotidiano da atenção básica. Corroborando isso, um estudo sobre a percepção acerca de sua identidade demonstra que a sua prática está associada à coordenação e possui valor devido a essa atribuição na equipe de ESF, reconhecida pelos demais membros da equipe. ${ }^{7}$

O enfermeiro toma para si a responsabilidade, incluindo os integrantes do matriciamento, os quais se sentem parte da equipe, sentindo-se vinculados aos demais. Essa inclusão contribui positivamente para o apoio matricial, pois o referido profissional reconhece a importância da ocorrência de intervenções realizadas de modo conjunto no campo da saúde. Destarte, o vínculo entre os trabalhadores é fortalecido, facilitando a entrada dos núcleos de apoio junto às equipes de referência, o que faz com que a atuação proceda de forma colaborativa e numa perspectiva interprofissional. ${ }^{20}$ Caso isso não ocorra, o apoio matricial encontra dificuldades para vincular-se às equipes apoiadas o que, por consequência, pode comprometer a atuação na atenção básica.

$\mathrm{Na}$ ESF, o enfermeiro exerce funções diversas, juntamente com os demais profissionais, e precisa trabalhar de forma interdisciplinar, assumindo a responsabilidade com a equipe e as pessoas assistidas, construindo o cuidado colaborativo e compartilhado no território. Assim, é importante que sejam desenvolvidas ações e práticas em saúde mental na atenção básica, articulando o cuidado com todos os trabalhadores, mediado pela figura do enfermeiro. ${ }^{9}$

Dessa forma, é preciso destacar a liderança exercida pelos enfermeiros na atenção básica, a qual compreende a dimensão gerencial do trabalho em enfermagem, sendo considerado como um elemento crítico e prioritário no trabalho em saúde. ${ }^{21}$ Ela precisa ser exercida com sabedoria e ciência, em virtude do profissional em questão absorver funções de gerenciamento e de assistência.

Um estudo realizado com enfermeiros da região sudeste brasileira demonstra que a 
complexidade da atenção básica requer que eles sejam qualificados para o desempenho das funções neste nível de atenção. É dado destaque, ainda, para algumas questões que atrapalham o desenvolvimento da liderança, como a falta de integração entre as equipes, a desmobilização do trabalho em saúde e a sobreposição de ações gerenciais e assistenciais. ${ }^{21}$

$\mathrm{Na}$ realidade estudada, foi possível visualizar a magnitude do trabalho do enfermeiro no contexto da atenção básica, observando as questões de gerenciamento e de liderança das equipes. Notou-se que algumas equipes estavam articuladas com o apoio matricial, utilizando a ferramenta e acessando os trabalhadores para fortalecer o trabalho na ESF. Ações viabilizadas pelo enfermeiro que facilita esse processo, por meio do exercício da liderança e do gerenciamento das equipes. Observou-se, mediante os dados apresentados, que o enfermeiro é uma liderança que agrega, problematizando situações, realizando cobranças necessárias e, ainda, praticando autocrítica, ou seja, reconhecendo as limitações e dificuldades encontradas para o trabalho na atenção básica.

É preciso que os enfermeiros assumam - com responsabilidade - a integração com as equipes de apoio matricial, uma vez que buscam, a partir disso, retaguarda especializada e suporte para tomada de decisão, especialmente, em casos complexos e de difícil resolução. Como exemplo, pessoas em sofrimento psíquico com dificuldade de adesão à proposta terapêutica, ou em situação em vulnerabilidade social, ou, ainda, sem suporte da rede familiar e social, dentre outras possibilidades. Percebeu-se que os aspectos relacionados à saúde mental são geralmente compartilhados com o apoio matricial. Assim, o cuidado compartilhado é uma estratégia amplamente difundida no contexto da assistência a condições crônicas, a partir da integração dos diferentes saberes. Também, pode ser entendido como cuidado integrado e colaborativo, o qual insere distintas disciplinas no mesmo ambiente de discussão. ${ }^{6}$

Outro entendimento da busca do apoio matricial para os casos de saúde mental pode estar relacionado à "falta de preparo" das equipes para trabalhar com essa realidade. Isso se deve, pois o sofrimento mental, muitas vezes, é visto de forma dissociada das demais condições crônicas. 
Contribuições do enfermeiro para o apoio matricial em saúde mental na atenção básica | 14

As condições crônicas vão, portanto, muito além das doenças crônicas [...], ao envolverem doenças infecciosas persistentes [...], condições ligadas à maternidade e ao período perinatal [...]; condições ligadas à manutenção da saúde por ciclos de vida [...]; distúrbios mentais de longo prazo; deficiências físicas e estruturais contínuas [...]; doenças metabólicas; doenças bucais; as condições de saúde caracterizadas como enfermidades (illnesses) em que há sofrimento, mas não doenças que se inscrevam nos padrões biomédicos. ${ }^{22: 27}$

Assim, é possível notar que apesar do sofrimento mental ser apresentado como condição crônica, há dificuldades em reconhecê-lo como tal, principalmente na atenção básica, na qual o modelo biomédico ainda impõe processos de trabalho fragmentados, configurando-se como barreira para que um cuidado integral seja viabilizado. Destaca-se o reconhecimento dos trabalhadores, em especial dos enfermeiros na atenção básica, de suas responsabilidades em relação ao cuidado de pessoas com diabetes, hipertensão, gestantes, puérperas e crianças, mas com certa dificuldade de reconhecer o sofrimento mental como essas condições. ${ }^{9}$

A partir disso, surge a necessidade de suporte para que os trabalhadores absorvam as demandas de saúde mental e atuem com esse olhar no cuidado prestado no território. Desse modo, o enfermeiro acolhe o apoio e percebe a necessidade de articulação para que as questões mais complexas sejam assistidas com suporte do matriciamento. Essa articulação é favorecida pela gestão do enfermeiro, que compreende a necessidade de contar com profissionais especializados e com uma ótica externa para trabalhar demandas complexas e, em especial, as de saúde mental.

Um estudo, com trabalhadores apoiadores e enfermeiros, voltado às ações de apoio matricial na atenção básica, identificou aspectos do cuidado em saúde mental no território. Apontou a necessidade de planejamento, integração e fortalecimento das ações realizadas pelas equipes, a fim de que a assistência seja qualificada, a autonomia das pessoas seja preservada, os trabalhadores estejam preparados e compartilhem o cuidado de forma interprofissional e interdisciplinar. ${ }^{23}$

Outro estudo realizado com enfermeiros da ESF e da atenção especializada em saúde mental no sudeste brasileiro indicou que o apoio matricial tem o intuito de ser "um caminho de esperança", 
no qual os atores colocam as melhores expectativas em relação à assistência às pessoas em sofrimento mental. Sinalizou, ainda, que o matriciamento é uma ferramenta potente na rede de atenção à saúde, especialmente, no cuidado à pessoa em sofrimento mental, pois os trabalhadores adicionam segurança em seu fazer cotidiano, sendo o apoio indispensável e essencial para que a assistência seja prestada com qualidade e resolutividade na atenção básica. ${ }^{10}$

Os gestores - participantes de um estudo, que analisou as estratégias, desafios e possibilidades da articulação entre a saúde mental e a atenção básica - sustentaram que o apoio matricial demanda uma relação dialógica entre os atores envolvidos, com base em uma lógica interprofissional e de uma prática colaborativa, buscando a integralidade do cuidado. ${ }^{20}$ Desta forma, compreender o apoio matricial a partir do diálogo e da contribuição das diferentes formações faz com que a atenção básica seja capaz de apostar nos cuidados em saúde mental no território. Cabe o destaque às diversidades existentes e a possibilidade de cuidado em liberdade, prezando pela autonomia, liberdade, cidadania e direitos humanos - princípios básicos da atenção psicossocial.

Atualmente, não se entende que os especialistas em saúde mental são, exclusivamente, os responsáveis pelos cuidados com as pessoas em sofrimento mental, pois todos os profissionais que atuam na atenção básica são corresponsáveis no processo de reabilitação psicossocial. O que facilita a atuação é a articulação da equipe multiprofissional, por meio da qual a resolutividade dos casos se torna viável, uma vez que os trabalhadores compartilham o mesmo objetivo. ${ }^{24}$

Os participantes deste estudo destacaram dificuldades no atendimento das situações de saúde mental, reconhecendo como uma área difícil de ser trabalhada, observando uma demanda crescente nas unidades de saúde e a falta de serviço especializado para encaminhamento. Porém, eles reconheceram a importância do cuidado em saúde mental, por meio da mobilização de ações junto ao apoio matricial e realização de diferentes intervenções no território.

Reforçando o que os participantes do presente estudo vivenciaram, uma pesquisa realizada com equipes de ESF no nordeste brasileiro investigou as percepções em relação às ações de saúde 
Contribuições do enfermeiro para o apoio matricial em saúde mental na atenção básica | 16

mental na atenção básica, destacando a importância de trabalhar com foco na saúde mental. Entretanto, reconheceu como dificuldade a grande demanda de atendimento, além da insegurança da maioria dos trabalhadores em viabilizar ações de saúde mental na atenção básica. ${ }^{25}$

Geralmente, municípios de pequeno porte não contam com serviços especializados em saúde mental, sendo a atenção básica é a responsável por esses casos, com suporte do apoio matricial e a colaboração dos demais serviços da rede. Assim, uma análise da organização da rede de atenção psicossocial, a partir das regiões de saúde do Brasil, detectou a existência de vazios assistenciais na saúde mental, sendo que existem municípios que contam apenas com dispositivos de atenção básica e não possuem nenhum outro ponto da rede de atenção psicossocial. Questão que preocupa no que diz respeito à expansão, à regionalização e ao movimento de interiorização da atenção psicossocial, caracterizando-se como limitação da universalização da cobertura de saúde mental do país. ${ }^{26}$

Assim, o apoio matricial atua na lógica de superação dos encaminhamentos, buscando desenvolver uma visão ampliada e integral do cuidado em saúde mental. Além de compreender a base dialógica da gestão, na construção do trabalho coletivo em saúde, priorizando o cuidado a pessoa e sua família. O matriciamento busca promover a discussão e viabilizar os modos possíveis de trabalhar na perspectiva da atenção psicossocial, do cuidado no território e respeitando as diferentes dimensões da pessoa em sofrimento mental, sua família e comunidade. ${ }^{27}$

Para fortalecer os princípios da Reforma Psiquiátrica e os pressupostos da atenção psicossocial, o apoio matricial é considerado como potente ferramenta de Educação Permanente em Saúde, a qual tem em suas bases referenciais pedagógicos inovadores e que atuam na construção de práticas integradoras e democráticas, como: aprendizagem significativa; processo de aprender a aprender; problematização; dentre outras. Consiste em uma proposta, com base nas metodologias ativas de ensino e aprendizagem, que coloca o cotidiano dos serviços e a formação profissional em constante análise, por meio da implicação dos sujeitos na transformação da realidade onde atuam. ${ }^{28}$ Assim, o enfermeiro apresenta papel na articulação, no estímulo e na execução de ações de 
Educação Permanente em Saúde, posto que, mediante práticas educativas significativas, os atores sociais se empoderam e transformam o seu trabalho. ${ }^{7}$ Além disso, o profissional em pauta privilegia o olhar, a reflexão e a intervenção sobre a realidade e as práticas do cotidiano dos serviços de saúde. ${ }^{8}$ Dessa maneira, o profissional de enfermagem atua na lógica de responsabilização dos trabalhadores pelo cuidado no território, transformando visões sobre a saúde mental e construindo estratégias participativas e colaborativas. ${ }^{23-24}$

Ao reconhecer que o cuidado com as pessoas em sofrimento mental na atenção básica precisa ocorrer de forma qualificada, discute-se a necessidade de formação dos trabalhadores para que seja viável uma assistência qualificada. Seguindo esse viés, é imperativo que o processo de Educação Permanente em Saúde transcorra de modo concreto e constante, com vistas ao cuidado integral às pessoas e suas famílias, com base no acolhimento e na escuta terapêutica. ${ }^{25}$ Assim, o apoio matricial pode colaborar com a dimensão didático-pedagógica, a qual é viabilizada pela construção de processos educativos, buscando qualificar o serviço e o cuidado em saúde mental. ${ }^{13}$

Contribuindo com esta discussão, um estudo com trabalhadores da ESF enfatizou a necessidade de investimentos na formação de enfermeiros no campo da saúde mental. Esses profissionais sentem-se inseguros na condução de casos dessa natureza, na estruturação de ações e estratégias para atendimento de pessoas em sofrimento psíquico. Com vistas à transformação desta realidade e com o objetivo de qualificar a assistência é que a Educação Permanente em Saúde precisa ser priorizada, rompendo com o paradigma vivenciado e buscando a consolidação do modelo de atenção psicossocial. ${ }^{10}$ Além disso, a ocorrência dos processos educativos, no dia a dia dos serviços, fortalecem as ações de cuidado, qualificando o trabalho em saúde e engendrando novas perspectivas de atuação no cotidiano profissional. ${ }^{28}$

Outro estudo do trabalho do enfermeiro na atenção básica revela que a inserção das equipes de ESF vem promovendo diferentes modos de produção do cuidado no território. Isso se deve, haja vista que, neste nível de atenção, as tecnologias leves recebem destaque e estão associadas à 
Contribuições do enfermeiro para o apoio matricial em saúde mental na atenção básica | 18

construção de vínculos, no campo das relações sociais, na escuta qualificada e no acolhimento. ${ }^{8}$ A partir disso, nota-se a importância do enfermeiro e de toda equipe de saúde aproximar-se da realidade do território no qual estão inseridos, conhecer as pessoas, suas necessidades e produzir, com elas, ações de melhoria do acesso e de construção de autonomia na comunidade.

Ao olhar para o cuidado em saúde mental se faz necessário compreender os tipos de tecnologias de cuidado, sejam elas leves, leve-duras e duras. As tecnologias leves são aquelas das relações, produção de vínculo, autonomização, acolhimento, gestão de processos de trabalho; as tecnologias leve-duras são os saberes bem estruturados que operam no trabalho em saúde como a clínica médica; e as duras são as relacionadas a equipamentos tecnológicos, normas, rotinas e estruturas organizacionais. ${ }^{29}$

Sobreleva-se as tecnologias leves como emblemáticas e fundamentais no cuidado em saúde mental, pois envolvem o campo das relações que produzem saúde e constroem autonomia com as pessoas. Assim, o vínculo articula-se com a satisfação da pessoa que é acompanhada, visto que se sente valorizada, com confiança no profissional e satisfeita com o atendimento recebido. Essas relações, que são estabelecidas entre o profissional de saúde e a pessoa assistida, demonstram a relevância que as relações intersubjetivas mantêm no cuidado e no trabalho em saúde. ${ }^{30}$ Enfatiza-se que conhecer o território, as pessoas, as realidades e compreendê-las em seu contexto, são fundamentais para construção do cuidado integral.

O presente artigo apresenta características de uma pesquisa qualitativa, como a singularidade, uma vez que se trata de um estudo realizado em apenas uma região de saúde, o que retrata uma realidade específica de um município do RS. Como limitação, pode-se salientar que esta investigação foi desenvolvida em um único local, apresentando dados de uma realidade específica. No entanto, as observações, as entrevistas, os grupos de validação e negociação e as discussões podem servir como indicativos para reflexões nesse cenário em novos estudos em outras especificidades, bem como outros contextos, a fim de ampliar o campo de investigação. Ressalta-se, 
com isso, a relevância do apoio matricial e da enfermagem como potencializadora dessas ações, no sentido de oferecer elementos para problematizar e discutir a saúde mental na atenção básica.

\section{Conclusão}

Este estudo oportunizou a identificação das contribuições do enfermeiro no contexto do apoio matricial em saúde mental na atenção básica. Tais contribuições podem ocorrer por meio da inclusão do matriciamento junto ao trabalho das equipes, pelo gerenciamento do serviço e pela liderança que contribuem na articulação, pelo cuidado compartilhado e pela mediação das ações de saúde mental no território.

A Enfermagem é uma profissão que concentra a atribuição do cuidado e, com isso, a corresponsabilidade pelas pessoas que acompanha. Notou-se, no contexto estudado como o enfermeiro realiza essa função, com o suporte do apoio matricial, inserindo os núcleos em seu fazer cotidiano, colaborando na construção de alternativas às pessoas em sofrimento mental, suas famílias e suas comunidades.

Além disso, observou-se que a liderança do enfermeiro na atenção básica é decisiva, pois concentra atribuições de gestão e de assistência. Assim, colabora para que os trabalhadores do apoio matricial, conjuntamente aos das equipes de referência, construam alternativas de cuidado no território, respeitando as individualidades das pessoas, construindo autonomia e liberdade.

Além dessas questões, algumas fragilidades foram visualizadas e apontadas pelos trabalhadores, como o aumento das demandas no campo da saúde mental e as dificuldades em relação à qualificação profissional nesta área. Isso elucida a necessidade de investimento nas ações de Educação Permanente em Saúde, para que os trabalhadores sejam empoderados para atuar com as questões de saúde mental na atenção básica.

Este estudo buscou contribuir com a atenção básica no sentido de oferecer suporte para o cuidado em saúde mental no território, suscitando a discussão desse tema a partir das contribuições 
do enfermeiro. Este, por sua vez, facilita o acesso do apoio matricial às equipes de ESF, sendo considerado como um destaque na questão de liderança nesse contexto e mantendo a abertura para o trabalho colaborativo e interprofissional na atenção básica. Assim, a profissão em pauta contribui decisivamente para a sustentabilidade do SUS, por meio do seu trabalho nos diferentes níveis e espaços de atuação. Sublinha-se a atuação na atenção básica, e é possível observar o quão importante a enfermagem se faz no contexto de cuidado em saúde mental no território.

\section{Referências}

1. World Health Organization (WHO). State of the world's nursing 2020: investing in education, jobs and leadership. Geneva: World Health Organization; 2020 [cited 2020 May 19]. Available from: https://apps.who.int/iris/bitstream/handle/10665/331673/9789240003293-eng.pdf

2. Machado MH. Perfil da enfermagem no Brasil: relatório final. Rio de Janeiro: NERHUS - DAPS ENSP/Fiocruz; 2017. 748 p.

3. Fundação Oswaldo Cruz (FIOCRUZ), Escola Politécnica de Saúde Joaquim Venâncio (EPSJV). Força motriz do SUS [Internet]. Rio de Janeiro: EPSJV; 2019 [acesso em 2020 maio 19]. Disponível em: http://www.epsjv.fiocruz.br/noticias/reportagem/forca-motriz-do-sus

4. Arcêncio RA. Nursing as the profession of the future and the foundation of universal health systems. Rev Latinoam Enferm. 2018;26:e3063. doi: https://doi.org/10.1590/1518-8345.0000.3063

5. Boff L. Saber cuidar: ética do humano - compaixão pela terra. 20ª ed. Petrópolis: Vozes; 2014.

6. Smith SM, Cousins G, Clyne B, Allwright S, O'Dowd T. Shared care across the interface between primary and specialty care in management of long term conditions. Cochrane Database Syst Rev. 2017;2:CD004910. doi: https://doi.org/10.1002/14651858.CD004910.pub3

7. Assis JT, Santos JF, Pinto LMC, Brito PKH, Ferreira MA, Fernandes MC. Identidade profissional do enfermeiro na percepção da equipe da estratégia saúde da família. Rev Saúde Ciênc [Internet]. 2018 [acesso em 2020 jan 08];7(3):43-58. Disponível em: https://rsc.revistas.ufcg.edu.br/index.php/rsc/article/view/141

8. Galavote HS, Zandonade E, Garcia ACP, Freitas PSS, Seidl H, Contarato PC, et al. The nurse's work in primary health care. Esc Anna Nery. 2016;20(1):90-8. doi: https://doi.org/10.5935/1414-8145.20160013

9. Nascimento MGG, Nadaleti NP, Vilela SC, Terra FS, Silva SA, Resck ZMR. Nursing work process in the promotion of mental health: reflective analysis. Rev Enferm Cent Oeste Min. 2017;7:e2097. doi: https://doi.org/10.19175/recom.v7i0.2097

10. Almeida DR, Soares JNC, Dias MG, Rocha FC, Andrade Neto GR, Andrade DLB. Care for carriers of mental disorder in primary care: an interdisciplinary and multiprofessional practice. Rev Pesq Cuid Fundam. 2020;12:420-5. doi: https://doi.org/10.9789/2175-5361.rpcfo.v12.8388 
11. Saraiva SAL, Zepeda J, Liria AF. Components of matrix support and collaborative mental health care: a narrative review. Ciênc Saúde Colet. 2020;25(2):553-65. doi: https://doi.org/10.1590/1413-81232020252.10092018

12. Castro CP, Campos GWS. Apoio matricial como articulador das relações interprofissionais entre serviços especializados e atenção primária à saúde. Physis (Rio J). 2016;26(2):455-81. doi: https://doi.org/10.1590/S010373312016000200007

13. Campos GWS, Domitti AC. Apoio matricial e equipe de referência: uma metodologia para gestão do trabalho interdisciplinar em saúde. Cad Saúde Pública. 2007;23(2):399-407. doi: https://doi.org/10.1590/S0102$311 X 2007000200016$

14. Araujo RCG, Santos RD, Mesquita KSF, Bento TMA, Silva LKB. Apoio matricial na atenção psicossocial com foco na enfermagem. Cad Graduação Ciênc Biol Saúde. [Internet]. 2019 [acesso em 2020 jun 02];5(2):20314. Disponível em: https://periodicos.set.edu.br/index.php/fitsbiosaude/article/view/6324/3343

15. Halcomb EJ, McInnes S, Patterson C, Moxham L. Nurse-delivered interventions for mental health in primary care: a systematic review of randomized controlled trials. Fam Pract. 2019;36(1):64-71. doi: https://doi.org/10.1093/fampra/cmy101

16. Guba EG, Lincoln YS. Avaliação de quarta geração. Campinas: Ed. UNICAMP; 2011. 320 p.

17. ESTADO DO RIO GRANDE DO SUL. Resolução nº 403/11 - CIB/RS, de 26 de outubro de 2011. Porto Alegre, RS: Secretaria de Saúde, 2011. Disponível em: http://www.saude.rs.gov.br/upload/arquivos/carga20170220/23102058-1340039277-cibr403-11.pdf. Acesso em: 13 jan. 2020.

18. Instituto Brasileiro de Geografia e Estatística (IBGE). IBGE Cidades [Internet]. 2019 [acesso em 2019 jun 28]. Disponível em: https://cidades.ibge.gov.br/

19. Cardano M. Métodos qualitativos para pesquisa em saúde. J Nurs Health. 2017;7(3):1-5. doi: https://doi.org/10.15210/JONAH.V7I3.12847

20. Souza AC, Amarante PD, Abrahão AL. Inclusion of mental health in primary health care: care strategy in the territory. Rev Bras Enferm. 2019;72(6):1677-82. doi: https://doi.org/10.1590/0034-7167-2018-0806

21. Oliveira C, Santos LC, Andrade J, Domingos TS, Spiri WC. Leadership in the perspective of family health strategy nurses. Rev Gaúcha Enferm. 2020;41:e20190106. doi: https://doi.org/10.1590/1983-1447.2020.20190106

22. Mendes EV. O cuidado das condições crônicas na atenção primária à saúde: o imperativo da consolidação da estratégia da saúde da família. Brasília (DF): Organização Pan-Americana da Saúde; 2012.

23. Oliveira GC, Schneider JF, Pinho LB, Camatta MW, Nasi C, Guimarães AN, et al. Matrix support in mental health in primary care: the vision of supporters and nurses. Rev Gaúcha Enferm. 2020;41(Spec No):e20190081. doi: https://doi.org/10.1590/1983-1447.2020.20190081

24. Gurgel ALLG, Jorge MSB, Caminha ECCR, Maia Neto JP, Vasconcelos MGF. Mental health care in the family health strategy: the experience of matrix support. Rev Enferm UERJ. 2017;25:e7101. doi: https://doi.org/10.12957/reuerj.2017.7101

25. Silva GR, Reis HFT, Dos-Santos EM, Souza MPA, Azevedo RL. Mental health in primary care: 
Contribuições do enfermeiro para o apoio matricial em saúde mental na atenção básica | 22

perceptions of the family health care team. Cogitare Enferm. 2016;21(2):1-7. doi: https://doi.org/10.5380/ce.v21i2.43861

26. Macedo JP, Abreu MM, Fontenele MG, Dimenstein M. A regionalização da saúde mental e os novos desafios da Reforma Psiquiátrica brasileira. Saúde Soc [Internet]. 2017 [acesso 2020 jun 01];26(1):155-70. Disponível em: https://www.scielo.br/pdf/sausoc/v26n1/1984-0470-sausoc-26-01-00155.pdf

27. Lima MC, Gonçalves TR. Apoio matricial como estratégia de ordenação do cuidado em saúde mental. Trab Educ Saúde. 2020;18(1):1-21. doi: https://doi.org/10.1590/1981-7746-sol00232

28. Gigante RL, Campos GWS. Política de formação e educação permanente em saúde no brasil: bases legais e referências teóricas. Trab Educ Saúde. 2016;14(3):747-63. doi: https://doi.org/10.1590/1981-7746-sip00124

29. Merhy EE. Saúde: a cartografia do trabalho vivo. 4를 ed. São Paulo: Hucitec; 2014.

30. Jorge MSB, Diniz AM, Lima LL, Penha JC. Matrix support, individual therapeutic project and prodution in mental health care. Texto Contexto Enferm. 2015;24(1):112-20. doi: https://doi.org/10.1590/010407072015002430013

Editora Científica Chefe: Cristiane Cardoso de Paula

Editor Associado: Daiana Foggiato de Siqueira

Fomento / Agradecimento: O presente trabalho foi realizado com apoio da Coordenação de Aperfeiçoamento de Pessoal de Nível Superior - Brasil (CAPES) - Código de Financiamento 001

\section{Autor correspondente}

Guilherme Emanuel Weiss Pinheiro

E-mail: enfermeiro.guipinheiro@gmail.com

Endereço: Avenida Roraima, 1000, Colégio Politécnico da UFSM, Prédio 70, Bloco G, Sala 321A.

CEP: $97105-900$

\section{Contribuições de Autoria}

1 - Guilherme Emanuel Weiss Pinheiro

Concepção ou desenho do estudo/pesquisa, análise e/ou interpretação dos dados, revisão final com participação crítica e intelectual no manuscrito.

\section{2 - Luciane Prado Kantorski}

Concepção ou desenho do estudo/pesquisa, análise e/ou interpretação dos dados, revisão final com participação crítica e intelectual no manuscrito.

\section{Como citar este artigo}

Pinheiro GEW, Kantorski LP. Nurses' contributions to matrix support in mental health in primary health care. Rev. Enferm. UFSM. 2021 [Accessed on: Year Month Day]; vol.11 e49: 1-22. DOI: https://doi.org/10.5902/2179769253339 
23 | Pinheiro GEW, Kantorski LP

Rev. Enferm. UFSM, Santa Maria, v11, e49: p. 1-22, 2021 